\title{
DEVELOPMENT OF VEHICLE TRACKING USING SENSOR FUSION
}

\author{
${ }^{1} M$ Hyndhavi, ${ }^{2}$ Dr. Sri Kavya \\ ${ }^{1}$ Student, K L University, India, hyndhavimettu@gmail.com \\ ${ }^{2}$ Professor, K L University, India, kavya@kluniversity.in
}

\begin{abstract}
The development of vehicle tracking using sensor fusion is presented in this paper. Advanced driver assistance systems (ADAS) are becoming more popular in recent years. These systems use sensor information for realtime control. To improve the standard and robustness, especially in the presence of environmental noises like varying lighting, weather conditions, and fusion of sensors has been the center of attention in recent studies. Faced with complex traffic conditions, the single sensor has been unable to meet the security requirements of ADAS and autonomous driving. The common environment perception sensors consist of radar, camera, and lidar which have both pros and cons. The sensor fusion is a necessary technology for autonomous driving which 1- Introduction

Tracking vehicles ahead are often done using radar and vision sensors independently to mix the strengths of every sensor and produce a strong and accurate estimate of the vehicle. Using the information with knowledge of uncertainties present in each sensor, an optimal estimate can be computed that combines the dynamics of the vehicle and detections for different sensors [1, 2]. Radar and vision sensors are inexpensive when compared with other sensors, which are widely used in tracking applications and therefore are considered for sensor fusion here. The strengths of one sensor are the weakness of the other, thus creating a scenario for the sensor fusion to be implemented [3]. The possibility of the multiple vehicles being tracked implies the need for a data association algorithm to separate each tracked object. For simulation purposes, testing out algorithms on MATLAB with the help of toolboxes may prove to be a better option.
\end{abstract}

\section{2- Sensor Fusion}

provides a better vision and understanding of vehicles surrounding. We mainly focus on highway scenarios that enable an autonomous car to comfortably follow other cars at various speeds while keeping a secure distance and mix the advantages of both sensors with a sensor fusion approach. The radar and vision sensor information are fused to produce robust and accurate measurements. And the experimental results indicate that the comparison of using only radar sensors and sensor fusion of both camera and radar sensors is presented in this paper. The algorithm is described along with simulation results by using MATLAB.

Keywords: Sensor fusion; advanced driver assistance systems (ADAS); autonomous cars; autonomous driving; radar and vision sensors.

It is the ability to bring together inputs from multiple radars, cameras to make a single model of the environment around a vehicle. The resulting model is more accurate because the strengths of the different sensors are balanced. Before using real-world experimental data, sensor fusion was attempted on synthetic data by creating lanes, vehicle objects, and realistic sensor models. Using the sensor fusion and automated driving toolbox, scenarios are often created with user-defined road profiles, vehicle properties, and sensor parameters. Sensor fusion using radar and vision sensors makes use of a data association algorithm to assign detections to tracks $[4,5,6]$.

\section{2-1- Multi-Sensor Data Fusion}

A single perception sensor is not enough to cover the high safety requirements of future vehicles. Thus, multiple sensors of different technologies have to be combined to accomplish redundancy and diversity. The sensor fusion system mitigates the weakness of the individual sensors and outputs a strong environment model in any scenario. The individual sensors work independently, causing 
the measurements from the sensors to arrive asynchronously. Additionally, since the sensors are typically mounted on different positions of the vehicle, each sensor has an individual point of view. The sensors require a common understanding of time, space, and a standardized data interface to perform sensor fusion [7, 8]. There are three types of sensor fusion architectures

i. Low-level sensor fusion

ii. Feature-level sensor fusion

iii. High-level sensor fusion

Low-level sensor fusion processes the raw data fetched from all individual sensors. The advantage is to process raw data obtained directly from the sensors, without any influence of data loss. The drawback is to handle the high amount of data and increased complexity to process. In feature-level sensor fusion, pre-processing is applied at the sensor level to extract certain features. These extracted features are then fused by the sensor fusion system. This architecture is a trade-off between the two other approaches. High-level sensor fusion performs the whole processing for each sensor individually. The object lists are then fused by the sensor fusion system.

The data from the different sensors have to be aligned both temporally and spatially to perform sensor fusion. The temporal alignment of the sensor data relies on accurate timestamps added to each measurement of the single sensors $[9,10]$. To achieve this, all involved units have to work with a perfectly synchronized global time base. For spatial alignment, the measurements from the different sensors have to be transferred into a common coordinate system.

\section{2-2- Sensor Properties}

The primary properties of the sensor are position, orientation, field of view (FOV), and resolution. Additional properties such as detection probability can also be set for higher fidelity modeling. FOV describes the scope of the sensor. It only detects objects inside its field of view. FOV is expressed in terms of azimuth angle and elevation angle. The angle from the $\mathrm{x}$-axis to the orthogonal projection of the vector onto the $x-y$ plane is defined as azimuth. The elevation is defined as the angle from the projection onto the $x-y$ plane to the vector [11 - 15]. Once these are defined, the corresponding resolutions can also be easily defined for the sensor.

\section{2-3- Multi-Object Tracker}

Tracking an object broadly consists of two steps. These are detection assignment and state estimation. For single object tracking detection, the assignment becomes relatively simple since we can neglect any surrounding objects. For multi-object tracking, data assignment becomes more involved especially when objects are close together. Complexity in state estimation remains relatively similar for single object and multiple objects [16 - 21].

Detection assignment is the process in which multiple sensor detections are analyzed and the best position detection is assigned to a specific object. An extended object may generate multiple detections for sensors with high resolution and therefore need to be clustered to get the best estimation results. State estimation is the process of estimating the state of a target object with information of the dynamic system and new sensor information. Since sensor detections may change abruptly depending on the actual state of the vehicle, the state estimation process should be able to filter out these changes and produce a smooth result [22-27].

\section{3- Methodology}

The use of sensor fusion generates a scenario and simulates sensor detections to track the vehicles. The main benefit of using scenario generation is the ability to create rare events and test the vehicle algorithms with them.

\section{3-1- Generate the Scenario}

The scenario generation is comprised of generating a road network, defining the moving vehicles. To test and create the ability of the sensor fusion to track a vehicle that is passing on the left of the ego vehicle and additional vehicles are in front of and behind the ego vehicle. Add $600 \mathrm{~m}$ road defined by using a set of points with two lanes, and each point defines the center of the road in 3-dimensional space [28, 29]. The passing car will start on the right lane then move to the left lane to pass, and return to the right lane. 
Simulate an ego vehicle that has six radar sensors and two vision sensors and has some overlap and coverage gap. It demonstrates the strength of vision sensors to accurately predict lateral position. Ego vehicle is equipped with a vision sensor and a long-range radar sensor on both the front and back of the vehicle. Each side of the vehicle has two short-range radar sensors covering 90 degrees each [30 - 33]. One sensor on each side covers from the middle of the vehicle to the forward and the other to the back. The radar and vision sensor parameters are

Table 1: Radar model parameters

\begin{tabular}{|c|c|c|}
\hline Radar parameters & Units & Values \\
\hline Update interval & Seconds & 0.1 \\
\hline Field of view & [azimuth, elevation] in \\
degrees & {$[20,5]$} \\
\hline Detection probability & - & 0.9 \\
\hline Azimuth resolution & Degrees & 10 \\
\hline Range resolution & Meters & 1.25 \\
\hline
\end{tabular}

Table 2: Vision model parameters

\begin{tabular}{|c|c|c|}
\hline Vision properties & Units & Values \\
\hline Update interval & Seconds & 0.1 \\
\hline Maximum range & Meters & 150 \\
\hline Detection probability & - & 0.9 \\
\hline False positives per image & - & 0.1 \\
\hline
\end{tabular}

\section{3-2- Supporting Functions}

To track the vehicles that are close to the ego vehicle a multi Object Tracker is created and the tracker uses the in it Sim Demo Filter function to initialize a constant velocity filter based on detections. To track the vehicle, radar sensor detections need an additional step before sending the measurement. A radar sensor with high resolution will produce multiple detections for a single object, thereby increasing the chance for duplicate tracks. This also increases the computational load on the data assignment algorithm. Detection clustering is used to alleviate this issue. The clustering function merges the multiple detections suspected to be of the same vehicle to single detection. Also, this function looks for detections that are closer than the size of a vehicle. Detections that fit the criterion are considered a cluster and are merged to a single detection at the centroid of the cluster. To represent the modified measurements noises the possibility that each detection can be anywhere on the vehicle.

\section{4- Results}

The proposed vehicle tracking algorithm using sensor fusion is simulated by MATLAB R2020a. In the results, we can see that the ego vehicle, a lead car in front of the ego vehicle, and a chase car behind the ego vehicle travels at $25 \mathrm{~m} / \mathrm{s}$ along the road. Another vehicle that travels at $35 \mathrm{~m} / \mathrm{s}$ along the road and passes the ego vehicle. The create Demo Display function creates a three-panel display are a top view, a chase-camera view that follows the ego vehicle, and a bird's-eye plot as shown in fig 1 , fig 2 , and fig 3 . 

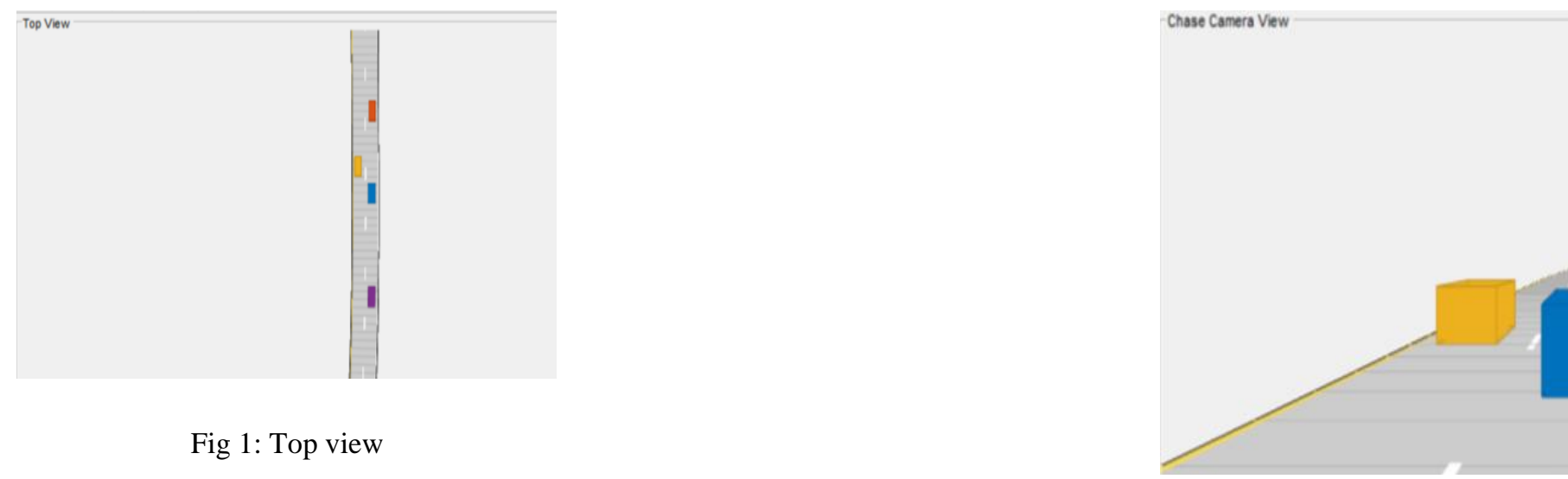

Fig 1: Top view

Fig 2: Chase camera view

All the radar and vision sensors cover 360 degrees field of view. Ego vehicle localization is carried out using lateral positioning from two vision sensors. In the bird's eye plot, it is visible the radar sensors, vision sensors, lane marking, and history up to 10 tracks. A function is used to update the bird's eye plot with road boundaries, detections, and tracks.

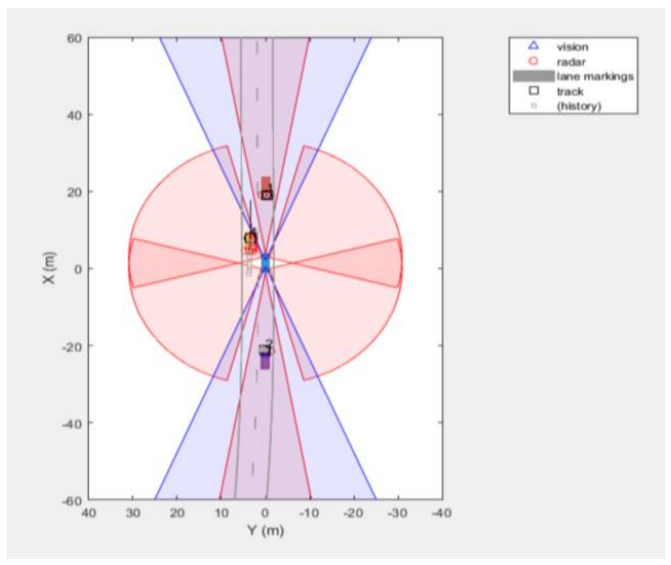

Fig 3: Bird's eye plot

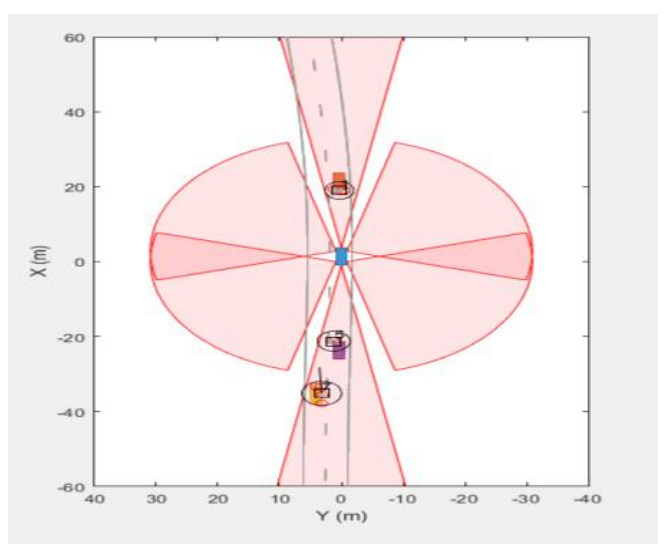

(a) 


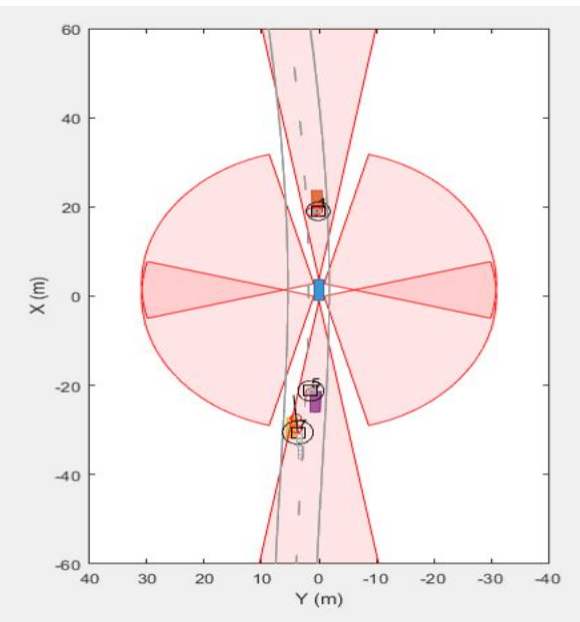

(b)

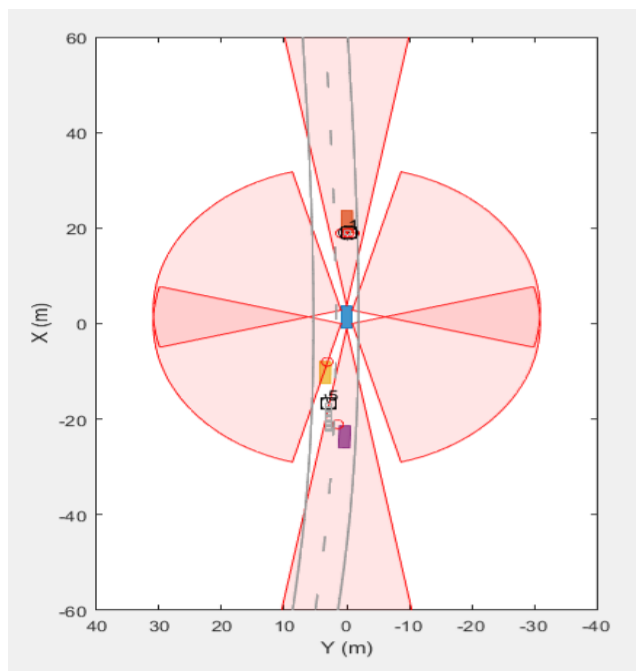

(c)

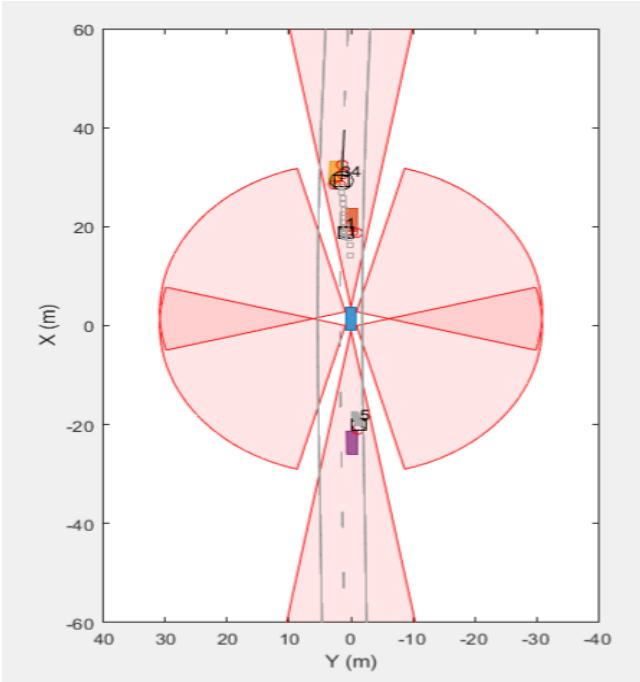

(d)

735 
Fig 4: Tracking of vehicles using six radar sensor

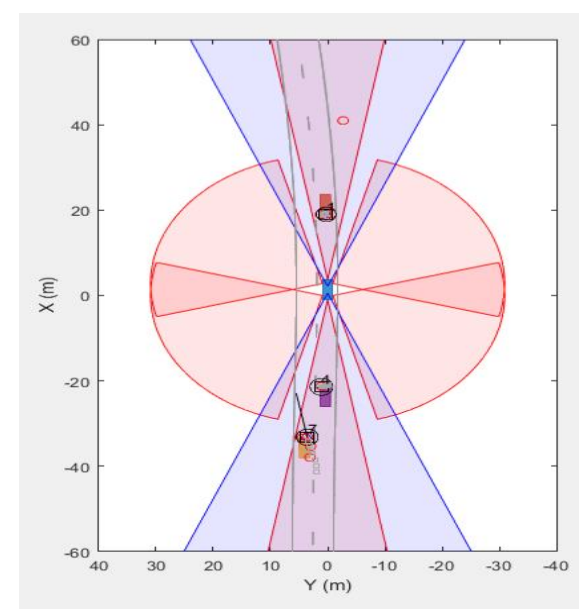

(a)

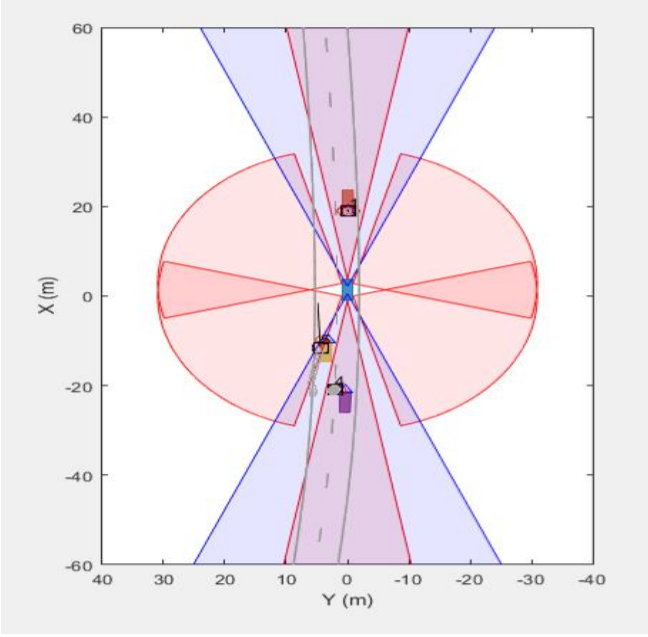

(b)

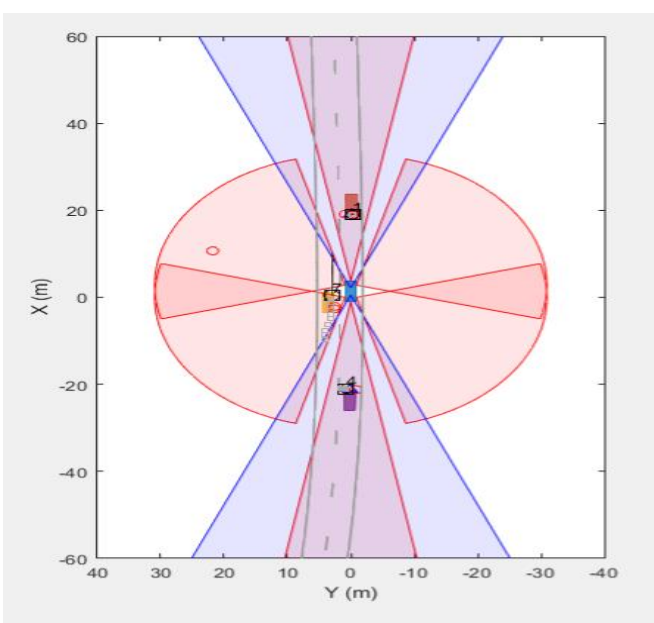

(c) 


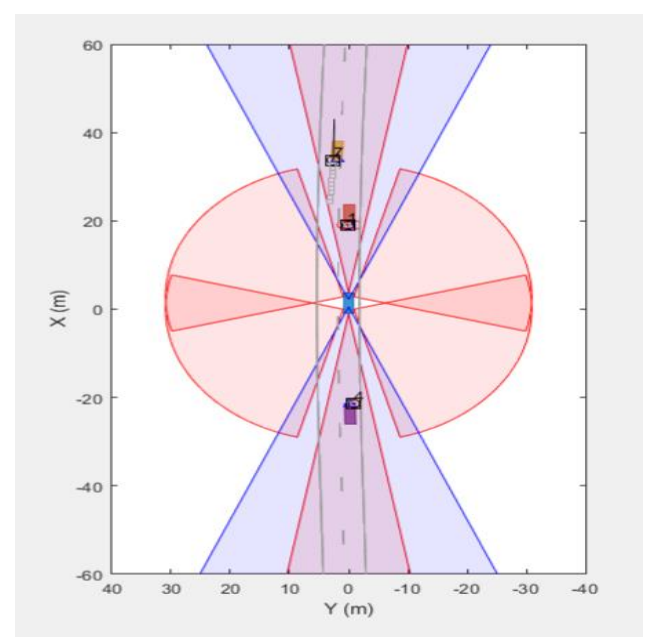

(d)

Fig 5: Tracking of vehicles using six radar and two vision sensor

From the above fig 4 and fig 5, the comparison of using radar sensors and sensor fusion of radar and vision sensors is shown in bird's eye plot. From the results, it is clear that when the radar sensors work standalone gives good detection of vehicles but poor in classification and color contrast. The sensor fusion of radar and vision sensors gives results that are more accurate.

\section{5- Conclusion}

In this paper, a method to fuse radar and vision sensor data has been described. The fused output shows good accuracy for both lateral and longitudinal positions of target vehicles. Radar sensors are accurate with longitudinal position tracking while vision sensors are accurate with lateral position tracking. A tracking algorithm is helpful to increase the robustness of the system and detection persistence. Camera-based technology provides information about color and appearance characteristics, but has limited performance under inclemency, low light conditions. Radar systems deliver reliable information about target position and velocity even in bad environmental conditions. Utilizing the full potential of radar sensors is still a big challenge of today. The obtained data from the sensor is significantly tougher to interpret than data from vision sensors. The simulation results show that the radar and camera sensor fusion can yield better performance and reasonable fusion results than the radar sensor tracking.

\section{References}

[1] E. Richter, U. Scheunert, F. Tango, and G. Wanielik. Advanced multiple objects tracking by fusing radar and image sensor information - applications. In Information Fusion, 11th International Conference on, July-2008, pages 1-7.

[2] H.cho, R. Rajkumar, Y.W. Seo, B.V. Kumar, "The multi-sensor fusion system for the moving object detections and tracking in the driving environment applications," in proceedings of (ICRA), 2014.

[3] B. Steux, C. Laurgeau, L. Salese and D.Wautier, Fade: vehicle detection and tracking system featuring monocular color vision and radar data fusion, IEEE Intelligent vehicles symposium proceedings, 2002.

[4] Omar. R, Garcia. C, Aycard. O. Classification for moving object detection and tracking and multiple sensor fusion. IEEE trans, 2016.

[5] H. Meinel, "For the autonomous driving the directions in the radar applications," in 2014.

[6] J.K. Suhr, D. Min, H.G. Jung, "The sensor fusion based vehicle localization system for urban environments," in IEEE Trans, 2017.

[7] Gerald Holweg, Christian Steger, N Druml, Josef Steinbeck, "Next-generation radar sensors in automotive sensor fusion systems" IEEE conference on sensor data fusion: Trends, Solutions, Applications 2017. 
[8] M. A. Richards, W. A. Holm, and W. L. Melvin. Principles of modern radar. Citeseer, 2010.

[9] Luca Bombini, Paolo Medici, and Giancarlo Aless. Radar-vision fusion for vehicle detection. In Procs. Intl. Workshop on Intelligent Transportation, pages 65-70, 2006.

[10] L. Walchshausl, R. Lindl, T. Tatschke, K. Vogel, "Detection of the road users in fused sensor information streams for the collision mitigation," in advanced systems for automotive applications 2006.

[11] Floudas N; Tsogas M; Polychronopoulos A; Amditis A. Multi-sensor coordination and fusion for automotive safety applications. In Proceedings of the International Conference on Information Fusion, Italy, July 2006, pp 10-13.

[12] P. Cerri, P. Medici, M. Bertozzi, L. Bombini, M. Miglietta, and P. Antonello: "Obstacle Detection and classification of fusing radar and vision sensors," in Intelligent Vehicles Conference, IEEE. June 2008.

[13] S.Sivaraman, M.Trivedi, "A survey of vision-based vehicle detection tracking and behavior analysis," IEEE Trans, Intell, Syst, Dec 2013.

[14] Guan H; Jia X; Wu Z: The Target Vehicle Movement State Estimation Technique with Radar supported Kalman Filtering Algorithm. In Proceedings of the International Symposium on Computer Communication Control and Automation, Singapore, Dec 2013; pp. 638-642.

[15] R. Mobus, U. Kolbe, "Multi-target tracking, the sensor fusion of radar and infrared," in Proceedings of IEEE Intelligent Vehicles Conference, 2004.

[16] Cheok, K.C.; Smid, G.E.; Mccune, D.J. A multi sensor-based collision avoidance system with application to a military. In Proceedings of the IEEE Intelligent Transportation Systems, October 2000; pp. 288-292.

[17] U. Franke, A. Gern, and P. Levi: "Sturdy vehicle tracking fusion of vision and radar." In Multi sensor Fusion and Integration for Intelligent Systems, 2001

[18] J. Laneurit, C. Blanc, and L. Trassoudaine, "The multi-sensory data fusion for the international vehicle and obstacle absolute positioning," IEEE Intelligent Vehicles Conference, June 2003, pp.138-143.

[19] Petrovskaya, A; Thrun, S: Model-based vehicle detection and tracking for autonomous urban driving, Springer, vol. 26, 2009.

[20] R.Faragher, "Understanding the basis of Kalman filter via easy and intuitive derivation," IEEE Trans 2012.

[21] H. Lee, H. Kim, B. Sung, "The multiple vehicle tracking and the estimation for comprehensive perception," in Proceedings of $12^{\text {th }}$ International symposium on advanced vehicle control, 2014.

[22] S. Ren, J. Sun. R-CNN: Towards real-time period object detection with region proposal networks. Advances in neural information systems, 2015, pages 91-99.

[23] Alkhorshid, Y: Camera-Based Lane Marking Detection for ADAS and Autonomous Driving, Springer, International Conference Image Analysis and Recognition - ICIAR 2015 .

[24] Xiao, F; Qin, B. A Weighted Combination Technique for Conflicting Proof in MultiSensor Data Fusion Sensors 2018.

[25] Wu, X., Ren, J., Wu, Y., and Shao, J., "Study on Target Tracking supported Vision and Radar Sensor Fusion," SAE Technical Paper 2018-01-0613, 2018.

[26] Ziebinski, A., Cupek, R., Erdogan, H., Waechter, S, "A Survey of ADAS Technologies for the Future Perspective of Sensor Fusion," In International Conference Computational Collective Intelligence.

[27] Garcia, F.; Martin, D.; La Escalera, A.D.; Armingol, J.M. Sensor Fusion Methodology for Vehicle Detection. IEEE Intell. Transp. System. Mag. 2017, 9, 123-133.

[28] Kim K, E Lee, and Lim: "The sensor fusion for vehicle tracking with the radar and camera sensors." In ICCAS - 17th International Conference on Control, Automation and Systems - 2017.

[29] Aeberhard, M. "Object-Level Fusion for the Surrounding Environment Perception in Automatic Driving Applications" Technische University of Dortmund: Germany, 2017. 
[30] Bahador Khaleghi, Alaa Khamis, Fakhreddine O. Karray, "Multi-sensory data fusion: Review of the state-of-the-art", Information Fusion, Volume. 14, No. 1, pp. 28-44, 2013.

[31] Lekic V, Babic Z. Automotive camera and radar fusion using Generative Adversarial Networks. Compute. Vis. Image Underst; 2019.

[32] K.Ch Sri Kavya, Sarat K Kotamraju, G Sree Teja: "Real time Signal Analysis for
Modelling the Performance of UHF/VHF Transceivers" In International Journal of Applied Engineering Research, Vol 9, 2014. Pages 8063 - 8088 .

[33] K.Ch. Sri Kavya, M. Ravi Kumar, Rahul: "Behaviour of Monopulse Radar" In International Journal of Computer Information Systems (IJCIS) Vol 3, 2012. Pages $18-22$. 